2007-01-01

\title{
Identification of a time-delayed process model using an overparameterisation method
}

John Roe

Technological University Dublin

Ruiyao Gao

Technological University Dublin

Aidan O'Dwyer

Technological University Dublin, aidan.odwyer@tudublin.ie

Follow this and additional works at: https://arrow.tudublin.ie/engscheleart

Part of the Controls and Control Theory Commons

\section{Recommended Citation}

Roe, John and Gao, Ruiyao and O'Dwyer, Aidan : Identification of a time-delayed process model using an overparameterisation method. Proceedings of the China-Ireland International Conference on Information and Communications Technologies (CIICT), DCU, August, 2007. doi:10.21427/rxag-8x14

This Conference Paper is brought to you for free and open access by the School of Electrical and Electronic Engineering at ARROW@TU Dublin. It has been accepted for inclusion in Conference papers by an authorized administrator of ARROW@TU Dublin. For more information, please contact arrow.admin@tudublin.ie, aisling.coyne@tudublin.ie, gerard.connolly@tudublin.ie.

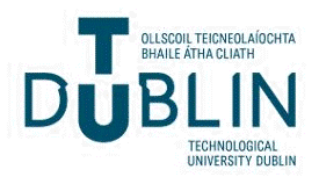




\title{
Identification of a time-delayed process model using an overparameterisation method
}

\author{
John Roe, Ruiyao Gao, Aidan O'Dwyer \\ School of Control Systems and Electrical Engineering, \\ Dublin Institute of Technology, \\ Kevin St., Dublin 8, Ireland \\ E-mail: aidan.odwyer@ dit.ie
}

\begin{abstract}
An extensive literature exists on the estimation of the model parameters of time-delayed processes. This paper briefly reviews the main overparameterisation methods for the Single-Input, Single Output (SISO) and Multi-Input, Multi-Output (MIMO) model parameter (and time delay) estimation problem. Subsequently, two algorithms, and their modifications, are discussed, and their performances are assessed in simulation. Results indicate that both the algorithms can suitably identify the model parameters, including the time delay. The third author has taught the method successfully to students taking advanced level control courses at DIT for a number of years; these experiences will be briefly reported. The paper also discusses the advantages and disadvantages of the overparameterisation approach.
\end{abstract}

Keywords: Identification, time-delay, overparameterisation

\section{Introduction}

On-line time delay estimation requires recursive estimation of the time delay in a closed loop environment; the method of overparameterisation is one such method. The method involves subsuming the time delay term (in the z-domain) into an extended (or overparameterised) numerator polynomial. The corresponding parameters are estimated using a recursive estimation scheme, and the time delay is calculated based on the parameters identified. The optimum value of the time delay corresponds to the smallest value of a quadratic loss function.

Assume that the process can be described by a linear difference equation (1) of order $n$ with constant parameters:

$$
\mathrm{y}(\mathrm{k})=-\mathrm{a}_{1} \mathrm{y}(\mathrm{k}-1)-\ldots-\mathrm{a}_{\mathrm{n}} \mathrm{y}(\mathrm{k}-\mathrm{n})+\mathrm{b}_{1} \mathrm{u}(\mathrm{k}-\mathrm{d}-1)+\ldots+\mathrm{b}_{\mathrm{m}} \mathrm{u}(\mathrm{k}-\mathrm{d}-\mathrm{m})+\mathrm{w}(\mathrm{k})
$$

with $\mathrm{u}(\mathrm{k})$ and $\mathrm{y}(\mathrm{k})$ being the sampled process input and output signals; $\mathrm{w}(\mathrm{k})$ is a white noise signal. The discrete process time delay index is $d=\tau / T$, where $d$ is a non-negative integer, $\tau$ is the time delay and $\mathrm{T}$ is the sampling time. The $\mathrm{z}$ transformation of equation (1) is:

$$
\mathrm{y}(\mathrm{z})=\frac{\mathrm{B}\left(\mathrm{z}^{-1}\right)}{\mathrm{A}\left(\mathrm{z}^{-1}\right)} \mathrm{z}^{-\mathrm{d}} \mathrm{u}(\mathrm{z})+\mathrm{w}(\mathrm{z})
$$

with $\mathrm{A}\left(\mathrm{z}^{-1}\right)=1+\mathrm{a}_{1} \mathrm{z}^{-1}+\ldots+\mathrm{a}_{\mathrm{n}} \mathrm{z}^{-\mathrm{n}}, \mathrm{B}\left(\mathrm{z}^{-1}\right)=\mathrm{b}_{1} \mathrm{z}^{-1}+\ldots+\mathrm{b}_{\mathrm{m}} \mathrm{z}^{-\mathrm{m}}$. The overparameterisation approach is based on the Recursive Least Squares (RLS) algorithm, in which equation (2) can be rewritten in a modified structure as follows:

$$
\mathrm{y}(\mathrm{z})=\frac{\mathrm{B}^{*}\left(\mathrm{z}^{-1}\right)}{\mathrm{A}\left(\mathrm{z}^{-1}\right)} \mathrm{u}(\mathrm{z})+\mathrm{w}(\mathrm{z})
$$

with 


$$
\mathrm{B}^{*}(\mathrm{z})=\mathrm{b}_{1}^{*} \mathrm{z}^{-1}+\ldots+\mathrm{b}_{\mathrm{m}+\mathrm{d}_{\max }^{*}} \mathrm{z}^{-\left(\mathrm{m}+\mathrm{d}_{\max }\right)}
$$

and $\mathrm{d}_{\max }$ is the upper limit of the process time delay index, which is assumed known a priori. For a process with the time delay index $\mathrm{d}$, the following equations hold:

$$
b_{i}^{*}=0, i=1,2 \ldots d ; b_{i}^{*}=b_{i-d}, i=d, \ldots m+d ; b_{i}^{*}=0, i=m+d+1, \ldots m+d_{\text {max }} .
$$

The parameters of the modified process model

$$
\hat{G}^{*}\left(z^{-1}\right)=\frac{\hat{B}^{*}\left(z^{-1}\right)}{\hat{A}^{*}\left(z^{-1}\right)}=\frac{\widehat{b}_{1}^{*} z^{-1}+\ldots+\widehat{b}_{m+d_{\max }}^{*} z^{-\left(m+d_{\max }\right)}}{1+\hat{a}_{1} z^{-1}+\ldots+\hat{a}_{n} z^{-n}}
$$

may be estimated using the RLS algorithm:

$$
\hat{\theta}(\mathrm{k}+1)=\hat{\theta}(\mathrm{k})+\gamma(\mathrm{k}) \mathrm{e}(\mathrm{k}+1)
$$

with

$$
\begin{gathered}
\gamma(\mathrm{k})=\mathrm{u}(\mathrm{k}+1) \mathrm{P}(\mathrm{k}) \phi(\mathrm{k}+1), \mathrm{e}(\mathrm{k}+1)=\mathrm{y}(\mathrm{k}+1)-\phi^{\mathrm{T}}(\mathrm{k}+1) \hat{\theta}(\mathrm{k}), \\
\hat{\theta}(\mathrm{k})=\left[\mathrm{a}_{1}(\mathrm{k}), \ldots \widehat{\mathrm{a}}_{\mathrm{n}}(\mathrm{k}), \hat{\mathrm{b}}_{1}^{*}(\mathrm{k}), \ldots \hat{\mathrm{b}}_{\mathrm{m}+\mathrm{d}}^{*}{ }_{\max }(\mathrm{k})\right], \\
\phi^{\mathrm{T}}(\mathrm{k})=\left[-\mathrm{y}(\mathrm{k}-1) . .-\mathrm{y}(\mathrm{k}-\mathrm{n}) \mathrm{u}(\mathrm{k}-1) . . \mathrm{u}\left(\mathrm{k}-\mathrm{m}-\mathrm{d}_{\max }\right)\right], \\
\mathrm{u}(\mathrm{k}+1)=\left[\lambda+\phi^{\mathrm{T}}(\mathrm{k}+1) \mathrm{P}(\mathrm{k}) \phi(\mathrm{k}+1)\right]^{-1}, \mathrm{P}(\mathrm{k}+1)=\left[\mathrm{I}-\gamma(\mathrm{k}) \phi^{\mathrm{T}}(\mathrm{k}+1)\right] \mathrm{P}(\mathrm{k}) / \lambda,
\end{gathered}
$$

$\lambda=$ forgetting factor, $\mathrm{I}=$ identity matrix.

For a noise free system, all numerator parameters whose indices are smaller than the time delay index should be identified as zero; this ideal situation does not apply in real applications. In addition, only values of the time delay that are integer multiples of the sample period are directly estimated by the method.

Many variations of the overparameterisation methods have been defined to calculate the numerator (and denominator) parameters, and subsequently the time delay, for processes that may be modelled in SISO form. Kurz [1] and Kurz and Goedecke [2], for example, define a robust method for estimating the SISO model parameters that is equivalent to determining the best match between the impulse response of the overparameterised model and the impulse response of a non-overparameterised model with a pure time delay; the method suffers from the disadvantage of having a heavy computational load. Other methods offer various trade-offs between robustness and computational load [3-28]. A recursive method to estimate the parameters, order and time delay index for both a stochastic system and a deterministic system, using an overparameterised method to estimate the time delay, is also described [29-31]. In interesting papers, Keviczky and Banyasz [32], [33] identify the time delay index using overparameterisation in the delta domain.

Other authors identify MIMO process models (with time delays) using the method of overparameterisation; Gurubasavaraj and Brogan [34], for instance, extend the method of Kurz and Goedecke [2] to estimate the time de lay for each input-output pair of a MIMO process model. Simulation results presented by the authors show that the time delays may be estimated in 20 sample periods, for a $2 \times 2$ MIMO process with a maximum time delay index of 4 ; the process order is, however, assumed known a priori. Other authors also use overparameterisation to identify MIMO process models [30], [35]. However, it is surprising that the overparameterisation methods that have been well documented for the estimation of the process time delay in the SISO environment have not been widely applied to the identification of time delays in MIMO processes in the available literature; the authors that use overparameterisation in the discrete-time MIMO environment [36-39] tend to use the resultant estimated parameters to define an appropriate control law, rather than to estimate the time delays.

In a previous paper [40], the second and third authors have simulated the methods of Kurz [1] and Teng and Sirisena [18], for the estimation of the model parameters and time delay index of a SISO process model. These methods are quite robust, with the method of Teng and Sirisena [18] being particularly interesting, because of its relative computational simplicity. The present paper will expand on the earlier work by reconsidering the estimation of the model parameters and time delay, both for SISO and MIMO applications. Starting with the estimation of all SISO model parameters, when the time delay is an integer multiple of the sample period, using the method of De Keyser [11], the method 
will be extended to the estimation of all model parameters when the time delay is not an integer multiple of the sample period. Subsequently, the method of Wong and Bayoumi [7], De Keyser [11] and modified versions of these methods, will be considered for the estimation of all model parameters of a SISO, Multiple-Input, Single-Output (MISO) and MIMO process. Space considerations mean that only results for the MIMO process will be reported. In preliminary work, the authors have also designed and simulated adaptive controllers based on estimation of the SISO process model parameters, when the time delay is an integer multiple of the sample period; for space reasons, this work will not be reported in the paper, but will be discussed at the conference.

\section{Overparameterisation methods considered}

Section 21 will consider the estimation of the parameters and time delay of a SISO process model, when the time delay is an integer multiple of the sample period. Section 22 will treat the same issue when the time delay is a real multiple of the sample period, with Section 23 dealing with the estimation of the parameters and time delay of a MIMO process model, when the time delay is an integer multiple of the sample period.

\subsection{SISO process model - time delay is an integer multiple of the sample period}

As mentioned, the methods of Wong and Bayoumi [7] and De Keyser [11] have been considered. Wong and Bayoumi [7] suggest that, as all numerator parameters whose indices are smaller than the time delay index are close to zero, though not exactly zero in practice, the correct time delay index value may be estimated as the index of the first parameter of $\hat{B}^{*}\left(z^{-1}\right)$ above a defined threshold level. A challenge is to define the threshold level, so this method best allows the estimation of an initial value of the (possibly time varying) time delay index. De Keyser [11] suggests that this initial value of time delay index may be improved by changing it according to the results of two inequality equations, which use the estimated model parameters as data:

if $\left|\hat{\mathrm{b}}_{\mathrm{d}}^{*}\right|>\mathrm{k}\left|\hat{\mathrm{b}}_{\mathrm{m}+\mathrm{d}+1}^{*}\right| \ldots$ decrease time delay index; if $\left|\hat{\mathrm{b}}_{\mathrm{d}}^{*}\right|<\left|\hat{\mathrm{b}}_{\mathrm{m}+\mathrm{d}+1}^{*}\right| / \mathrm{k} \ldots$ increase time delay index, $\mathrm{k}=5$. The following process model is identified in simulation:

$$
\mathrm{G}\left(\mathrm{z}^{-1}\right)=\frac{0.6+0.3 \mathrm{z}^{-1}}{1-0.4 \mathrm{z}^{-1}-0.32 \mathrm{z}^{-2}} \mathrm{z}^{-\mathrm{d}}
$$

d varies every 150 sample periods from 2 to 1 to 2 to 3 to 4 . Thus, the overparameterised model is

$$
G^{*}\left(z^{-1}\right)=\frac{b_{1} z^{-1}+b_{2} z^{-2}+b_{3} z^{-3}+b_{4} z^{-4}+b_{5} z^{-5}}{1+a_{1} z^{-1}+a_{2} z^{-2}}
$$

The parameters of this model are estimated using the U-D factorisation implementation of the RLS algorithm; a PRBS input is applied. Random noise of a maximum value of $\pm 10 \%$ of the signal level is added to the output of the difference equation that implements the model, to simulate a realistic scenario. The changing parameter values are tracked well by the algorithm, as shown in Figures 1a to $1 \mathrm{~g}$. These results are obtained when the algorithm is programmed in $\mathrm{C}$; similar results are also obtained using MATLAB.

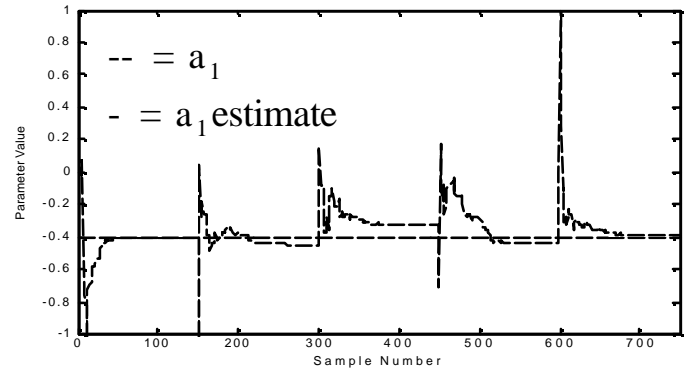

Figure 1a: $a_{1}, a_{1}$ estimate

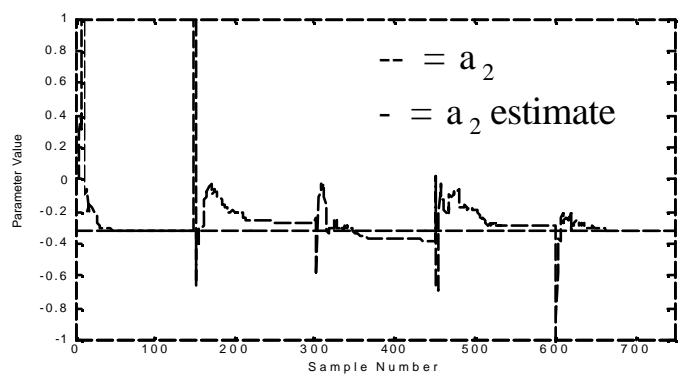

Figure 1b: $a_{2}, a_{2}$ estimate 


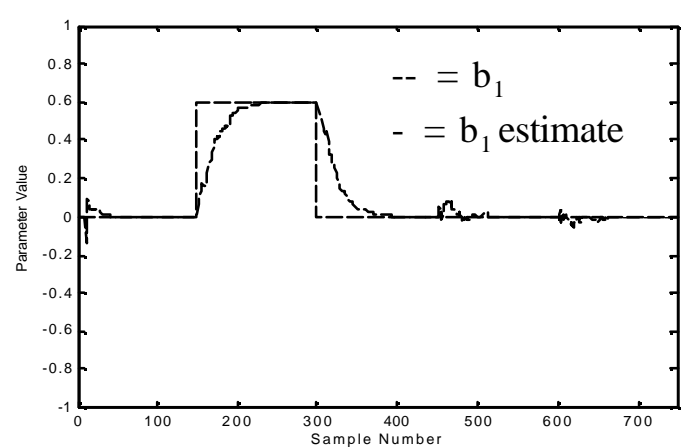

Figure 1c: $b_{1}, b_{1}$ estimate

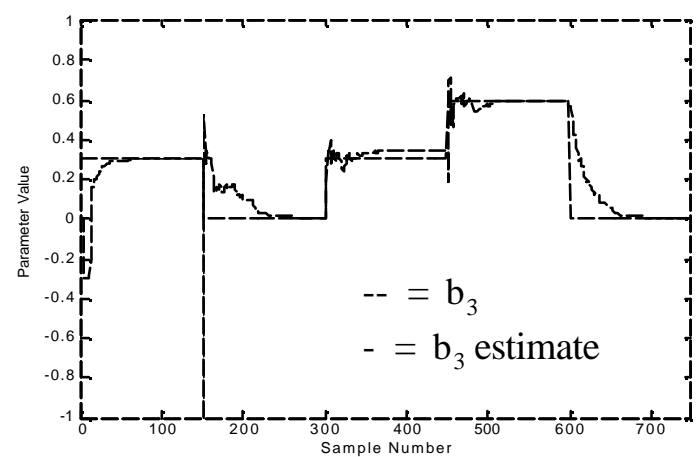

Figure 1e: $b_{3}, b_{3}$ estimate

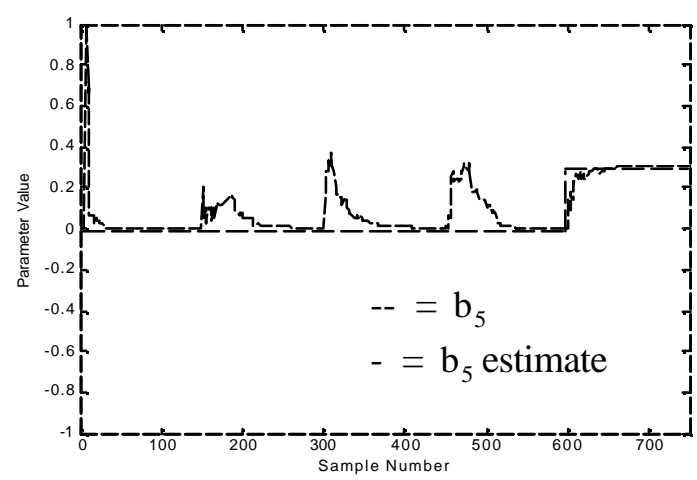

Figure 1g: $b_{5}, b_{5}$ estimate

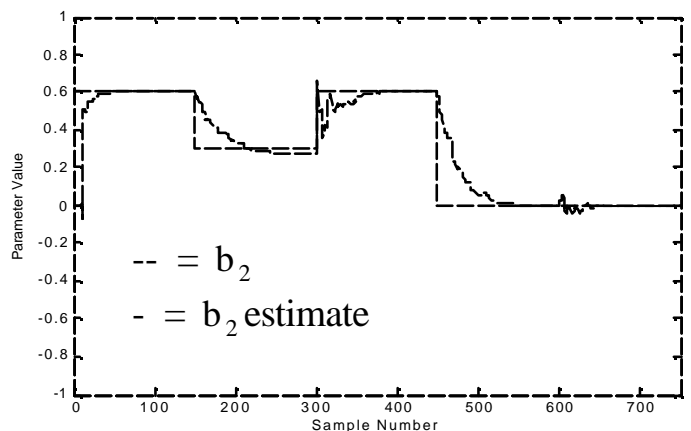

Figure 1d: $b_{2}, b_{2}$ estimate

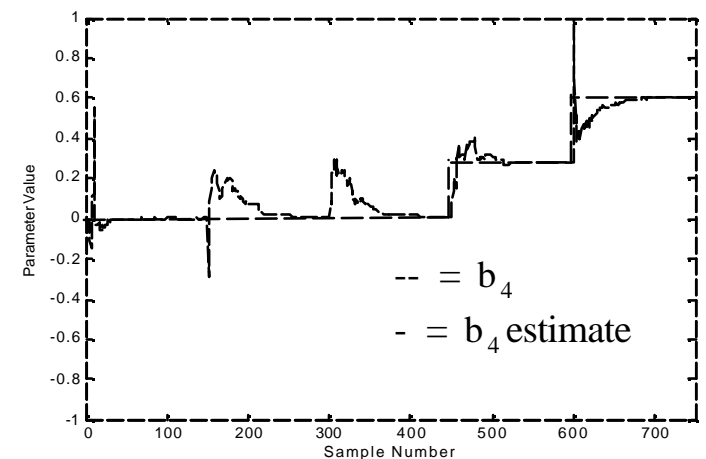

Figure 1f: $b_{4}, b_{4}$ estimate

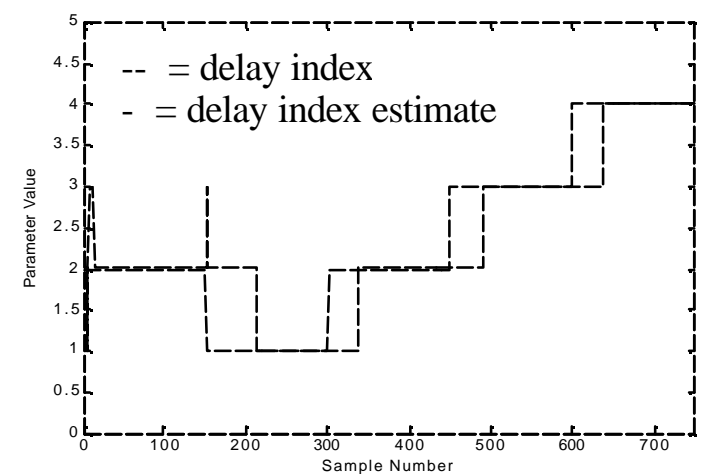

Figure 2: Delay index, delay index estimates

Once the parameters are estimated, the method of Wong and Bayoumi [7] is used to estimate the starting value of the time delay index; subsequently, the method of De Keyser [11] is used to track the variations in the time delay index. The result is shown in Figure 2. In this figure, $\mathrm{k}=5$, following the advice of De Keyser [11]; other results show satisfactory tracking when $\mathrm{k}=2.5,10$ and 20, with $\mathrm{k}=$ 2.5 facilitating the fastest tracking.

\subsection{SISO process model - time delay is a real multiple of the sample period}

The method of Wong and Bayoumi [7] may be expanded to estimate time delays that are non-integer multiples of the sample period (together with the process model parameters). Suppose the process in the continuous time domain is modelled in first order lag plus delay (FOLPD) form:

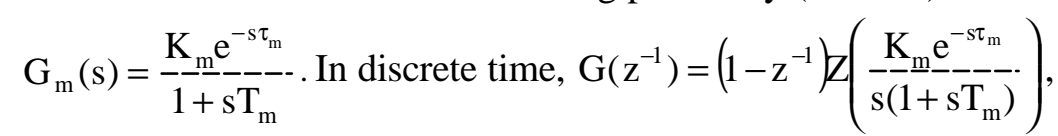

with $\tau_{\mathrm{m}}=\mathrm{dT}-\mathrm{fT}, \mathrm{d}=$ integer, $\mathrm{f}=$ fraction. It is straightforward to show that 


$$
G\left(z^{-1}\right)=\frac{\left[K_{m}\left(1-e^{-\frac{f T}{T_{m}}}\right)\right] z^{-d-1}+\left[K_{m}\left(e^{-\frac{f T}{T_{m}}}-e^{-\frac{T}{T_{m}}}\right)\right] z^{-d-2}}{1-e^{-\frac{T}{T_{m}}} z^{-1}}
$$

Identification of the parameters of $\mathrm{G}\left(\mathrm{z}^{-1}\right)$ will allow $\mathrm{f}$ to be calculated. The method may be expanded straightforwardly to estimate the delay when the model is in higher order form.

Two simulated FOLPD process models are identified (in the absence of noise). In both process models, $K_{m}=2, T_{m}=1$. In the first model, the time delay index, $d$, varies every 150 samples from 1.6 to 0.6 to 1.7 to 2.5 to 3.5 . Figure 3 shows the tracking result. In the second model, $d$ varies from 1.6 to 0.2 to 1.9 to 2.3 to 3.1 . Figure 4 shows the tracking result. The method is programmed in MATLAB; the System Identification toolbox is required. A PRBS input is applied to the simulated process, and the parameters of the process model are determined using the RLS algorithm. Space considerations mean the tracking of the non-delay process model parameters are omitted.

The simulation results show that the method of Wong and Bayoumi [7] may be expanded to estimate time delays that are multiples of 0.1 times the sample period. Other results show that the method of De Keyser [11] may also be successfully expanded.

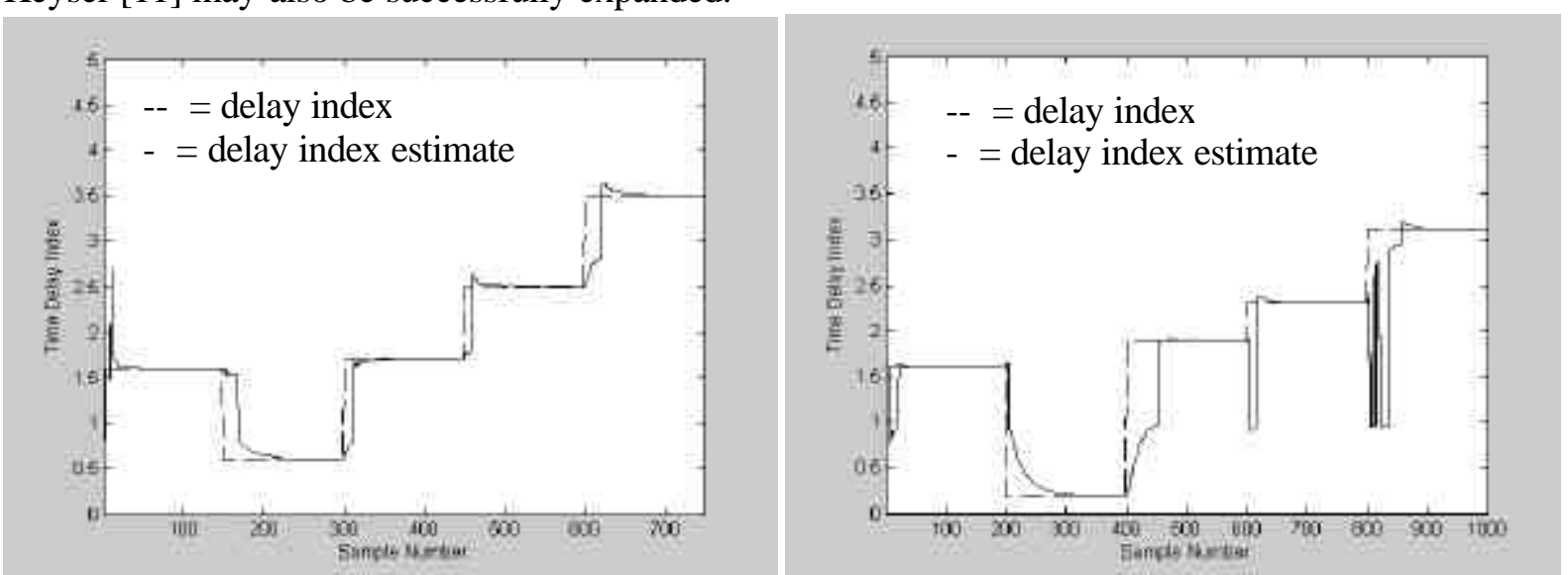

Figure 3: Delay index, delay index estimates

Figure 4: Delay index, delay index estimates

\subsection{MIMO process model - time delay is an integer multiple of the sample period}

Finally, the methods of Wong and Bayoumi [7] and De Keyser [11] may be expanded straightforwardly to estimate the time delay index of each element of a 2x2 MIMO process model, together with the other process model parameters. Each such element is modelled in FOLPD form; Wood and Berry [41], for example, develop a 2x2 MIMO model of this form for a distillation column. In a simulation, the MIMO process model from 0 to 300 sample periods, and from 300 to 600 sample periods is, respectively,

$$
\mathrm{G}_{\mathrm{m}}\left(\mathrm{z}^{-1}\right)=\left[\begin{array}{cc}
\frac{0.3544 \mathrm{z}^{-2}}{1-0.9419 \mathrm{z}^{-1}} & -\frac{0.3906 \mathrm{z}^{-4}}{1-0.9535 \mathrm{z}^{-1}} \\
\frac{0.45 \mathrm{z}^{-4}}{1-0.8765 \mathrm{z}^{-1}} & -\frac{0.2 \mathrm{z}^{-6}}{1-0.9415 \mathrm{z}^{-1}}
\end{array}\right], \mathrm{G}_{\mathrm{m}}\left(\mathrm{z}^{-1}\right)=\left[\begin{array}{cc}
\frac{0.3068 \mathrm{z}^{-6}}{1-0.9123 \mathrm{z}^{-1}} & -\frac{8.2096 \mathrm{z}^{-3}}{1-0.9329 \mathrm{z}^{-1}} \\
\frac{0.67 \mathrm{z}^{-3}}{1-0.9529 \mathrm{z}^{-1}} & -\frac{1.0 \mathrm{z}^{-4}}{1-0.9845 \mathrm{z}^{-1}}
\end{array}\right] .
$$

The elements of the MIMO process model terms are the discrete time equivalents of FOLPD continuous-time transfer functions.

As before, the method is simulated in MATLAB, using the System Identification toolbox. Independent PRBS inputs are applied to each element of the process model, and the parameters of each element are determined using the RLS algorithm. Random noise of a maximum value of $\pm 25 \%$ of the signal level is added to the output of the difference equation that implements the model.

Figures $5 \mathrm{a}$ to $5 \mathrm{~d}$ show the successful estimation and tracking of the changing time delay index value. Space considerations mean that the successful tracking of the non-delay process model parameters are omitted. 


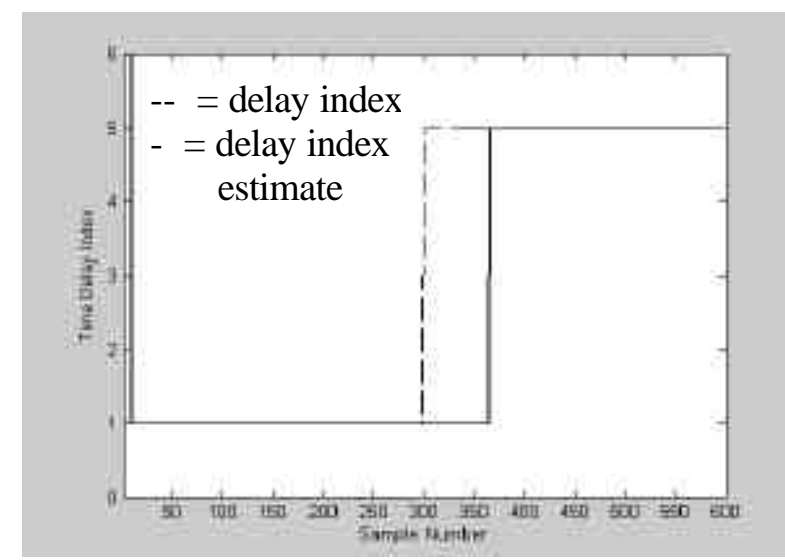

Figure 5a: Delay index estimate $-1 \mathrm{x} 1$ term

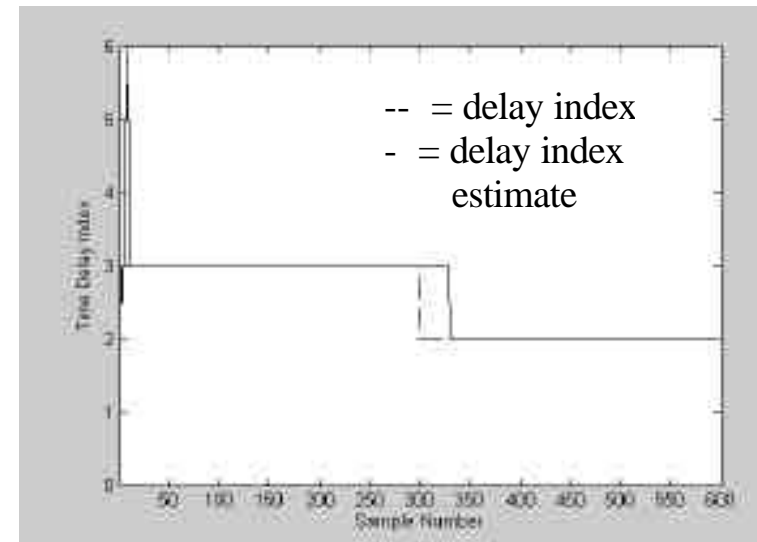

Figure 5c: Delay index estimate $-2 \mathrm{x} 1$ term

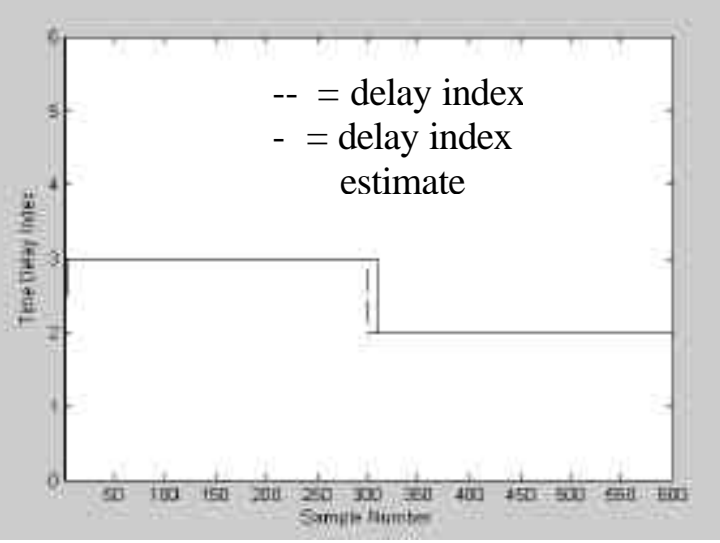

Figure 5b: Delay index estimate $-1 \times 2$ term

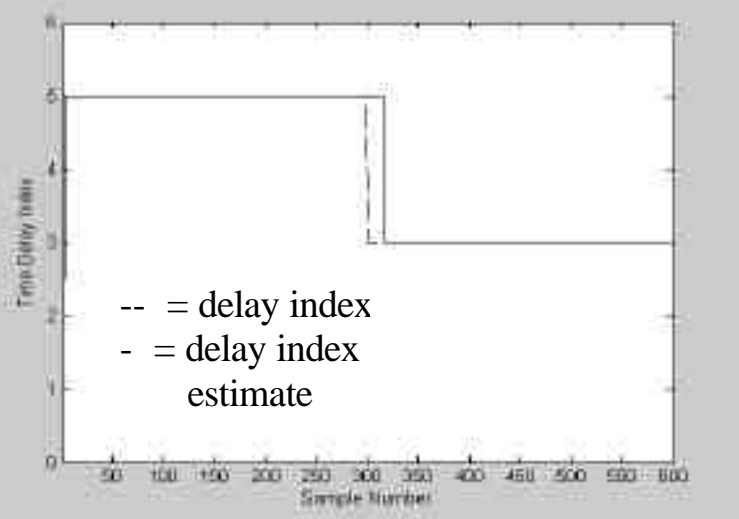

Figure 5d: Delay index estimate $-2 \times 2$ term

\section{Pedagogical Issues}

Teaching system identification and subsequently self-tuning control to undergraduate students has always been challenging, partly because of the mathematical preliminaries that are traditionally required. The topic has formed part of the Control Engineering syllabus of the honours B.E. in Electrical/Electronic Engineering at D.I.T. for at least 15 years, so that significant experience of effective teaching of the topic has been gained. However, in line with worldwide trends, time available on the programme for classroom and laboratory work has been reduced, in response to pressure to reduce class contact hours and the increasing desire to facilitate student self-learning. In this new environment, suggestions for relevant learning material and appropriate teaching methods are available [42-44]; however, typically twelve teaching hours is required [42]. On the honours B.E. programme, the total instruction time available for system identification and subsequent design of a PID self-tuning regulator is three hours. Thus, the third author has developed a teaching approach in which batch least squares engineering applications are introduced in the first hour. Subsequently, system identification case studies in MATLAB using recursive least squares, including the ones reported in Sections 2.1 and 2.2, are preformed for the second hour. The time consuming theoretical background is thus not taught, though the experience of the author is that little is consequently lost; the teaching method used does facilitate student self-learning and enhances understanding. In the final hour, the parameter estimates are used to explicitly design a PID self-tuning controller, following the method of Banyasz and Keviczky [45]. The author has not been able to formally measure the change in student learning quality, compared to a more traditional approach in which the recursive estimation algorithm is explored analytically in detail; however, the teaching approach is the most practical in the time available.

\section{General comments on the method of overparameterisation}

The attractiveness of the method of overparameterisation as a means of estimating model parameters 
and time delay is that it is a natural extension of methods used in delay-free identification applications. However, the method has a number of disadvantages.

(1) The computational burden of the RLS algorithm increases with the square of the number of estimated parameters [11], [46], [47].

(2) The persistent excitation condition (a condition for parameter convergence) is more difficult to satisfy for overparameterised models [48], [49].

(3) The adaptive capability of the corresponding controller is degraded, as it takes a long time for the parameters to be retuned if a change in the process dynamics occurs [48]. However, it is possible by introducing a perturbation signal into the regressor vector, when the parameters of the model with delay are being estimated, to achieve a similar convergence rate for the parameters of an overparameterised model as for the parameters of a non-overparameterised model [50], [51].

(4) The presence of a high order numerator polynomial increases the likelihood of common factors in the numerator and denominator polynomials in the estimation model, rendering identification more difficult [49].

(5) The overparameterisation method is not robust if a load disturbance is present, or if measurement noise is significant [52]. However, it is stated [50], [51] that injecting an excitation signal into the regressor vector (for RLS estimation [50] or recursive extended least squares (RELS) estimation [51]) allows the parameters of a model of one order of overparameterisation [50] or arbitrary degree of overparameterisation [51] to have the same guaranteed convergence as the parameters of a non-overparameterised model (i.e. ill-conditioning is avoided for the overparameterised model) for both models with white noise excitation [50] and coloured noise excitation [51].

From this discussion, the biggest disadvantage of the overparameterisation method for the identification of a process with time varying delay in closed loop, perturbed by a pseudo-random binary signal (PRBS), is the extra computational burden associated with identifying a greater number of numerator parameters. In an attempt to reduce the computational burden associated with the overparameterisation method, the following ideas may be worth considering in future work:

(a) If the time constants of the process do not change significantly, then the denominator parameters need not be estimated on-line [53]; as well as reducing the computational burden in the estimation stage, other advantages of this scheme are that excessive fluctuation of the denominator parameters is avoided and the denominator parameter estimates cannot drift into or near an undesirable region. A further suggestion [54] is that selective updating of certain model parameters be employed when the number of parameters of the process to be estimated is large; such selective updating could be achieved by only updating those parameters that give a significant improvement in the residual of the model fit.

(b) The sampling interval could be adapted to reduce the number of parameters to be estimated. To this end, it is suggested [54] that the sampling period be chosen so that the time delay index has a value of two or three; such slow sampling, the authors suggest, has the additional advantage of increasing the robustness of the corresponding adaptive controller. This advice may be relevant only for small values of the time delay as otherwise it may conflict with the most often quoted rule of thumb that the sampling period should be between one fifth and one fifteenth of the $95 \%$ rise time of the process step response [56].

\section{Conclusions}

The paper has briefly surveyed the range of overparameterised algorithms to estimate model parameters and the time delay of processes modelled in SISO and MIMO form, in the discrete time domain. Subsequently, two algorithms are described for parameter estimation of SISO process models, and a simulation result, showing successful estimation of the parameters when the time delay is an integer multiple of the sample period, using the method described by De Keyser [11], is given. It is shown that overparameterisation methods may be expanded to allow estimation of the parameters of process models where the time delay is not an integer multiple of the sample period. Subsequently, it is shown in simulation that the methods of Wong and Bayoumi [7] and De Keyser [11] may be expanded to allow estimation of the time delay indices (and, indeed, the other model parameters) of each element of a $2 \times 2$ MIMO process model; the time delays are integer multiple of the sample periods. The research work has directly influenced the third authors teaching of system identification, 
details of which are provided. Advantages and disadvantages of overparameterisation methods are also discussed, with suggestions made as to how any overparameterisation method may be improved.

Further work will concentrate on applying the methods to the estimation of parameters of real processes, including processes modelled in MIMO form where the time delay is not an integer multiple of the sample period. In addition, the author proposes to consider further the use of the delta operator rather than the shift (z) operator; some work [56], [57] has suggested that the delta operator reduces coefficient sensitivity problems (at higher sampling frequencies) and allows superior finite word length coefficient representation, though the operator may slow the convergence of parameter estimates, when compared to the shift operator.

\section{References}

[1] Kurz, H. (1979). Digital parameter-adaptive control of processes with unknown constant or timevarying dead time. Proc. Fifth IFAC Symposium on Identification and Parameter Estimation, 1187-1194.

[2] Kurz, H. and Goedecke, W. (1981). Digital parameter-adaptive control of processes with unknown dead time. Automatica, 17:245-252.

[3] Lammers, H.C. and Verbruggen, H.B. (1985). Simple self-tuning control of processes with a slowly varying time delay, Proc. IEE Control Conference, 393-398.

[4] Biswas, K.K. and Singh, G. (1978). Identification of stochastic time delay systems. IEEE Transactions on Automatic Control, AC-23:504-505.

[5] Astrom, K.J. and Zhou, Z.-Y. (1981). Self-tuners with automatic adjustment of the sampling period for processes with time delays. Report, Department of Automatic Control, Lund Institute of Technology .

[6] Friedlander, B. (1980). An ARMA modeling approach to multitarget tracking. Proc. 19th IEEE Conference on Decision and Control, 820-825.

[7] Wong, K.Y. and Bayoumi, M.M. (1982). A self-tuning control algorithm for systems with unknown time delay. Proc. IFAC Identification and System Parameter Estimation Conference, 1193-1198.

[8] Habermayer, M. and Keviczky, L. (1985). Investigation of an adaptive Smith controller by simulation. Proc. IFAC Seventh Conference on Digital Computer Applications to Process Control, 373-377.

[9] Habermayer, M. (1986). Quality investigation of an adaptive Smith predictor. Simulation of Control Systems: Selected Papers from the IFAC Symposium, 211-215.

[10] Batur, C. (1986). Stable sub-optimum controllers for the Smith dead-time compensation. Proc. American Control Conference, $2: 1354-1357$

[11] De Keyser, R.M.C. (1986). Adaptive dead-time estimation. Proc. IFAC Adaptive Systems in Control and Signal Processing Conference, 385-389.

[12] Koivo, H., Virtanen, P. and Pusenius, M. (1988). A self-tuning controller for processes with time varying delay: application to a paper machine head box. Proc. IFAC Adaptive Control of Chemical Processes Conference, 125-128.

[13] $\mathrm{Hu}$ X., Ng, T.S. and Xu H. (1988). Robust self tuner for variable time delay systems. Proc. Eighth IFAC/IFORS Symposium on Identification and System Parameter Estimation, 245-249.

[14] Keviczky, L. and Banyasz, Cs. (1988). A completely adaptive PID regulator. Proc. IFAC Identification and System Parameter Estimation Conference, 89-95.

[15] Najim, K., Youlal, H. and Najim, M. (1988). Identification of a pulsed liquid-liquid extraction column. Selected papers from the 8th IFAC/IFORS Symposium on Identification and System Parameter Estimation Conference, 1165-1170.

[16] Peter, K. and Isermann, R. (1988). Predictive self-tuning control of a thermal process with unknown deadtimes, Proc. IFAC Adaptive Control of Chemical Processes Conference, 69-74.

[17] $\mathrm{Xu}, \mathrm{H}$. (1988). Robust protection-time delay tracing adaptive control and its application. Proc. Eighth IFAC/IFORS Symposium on Identification and System Parameter Estimation, 769-774.

[18] Teng, F.-C. and Sirisena, H.R. (1988). Self-tuning PID controllers for dead time processes. IEEE Transactions on Industrial Electronics, 35:119-125.

[19] Thomson, M., Cassidy, P.G. and Sandoz, D.J. (1989). Automatic tuning of PID controllers using a combined time- and frequency-domain method, Transactions of the Institute of Measurement and Control, 11:40-47.

[20] Landau, I.D. (1990). System Identification and Control Design, Prentice-Hall Inc.

[21] Teng, F.-C. (1990). Investigation of the enlarged least-square estimator combined with a classical PID controller. Transactions of the Institute of Measurement and Control, 12: 224-228.

[22] Guez, A. and Piovoso, M. (1991). Custom neurocontroller for a time delay process. Proc. American Control Conference, 2:1592-1596.

[23] Isermann, R., Lachmann, K.-H. and Matko, D. (1992). Adaptive Control Systems, Prentice-Hall International Series in Systems and Control Engineering.

[24] Lundh, M. and Astrom, K.J. (1994). Automatic initialisation of a robust self-tuning controller. Automatica, 30:16491662.

[25] Readle, J.C. and Henry, R.M. (1994). On-line determination of time delay using multiple recursive estimators and fuzzy reasoning. Proc. International Conference on Control, 2:1436-1441.

[26] Disdell, K.J., Burnham, K.J. and James, D.J.G. (1995). Intelligent switching software for variable dead-time compensation within self-tuning control, Proc. $11^{\text {th }}$ IASTED International Conference in Applied Informatics, $289-292$. 
[27] Makoudi, M. and Radouane, L. (1999). Robust decentralized adaptive control for non-minimum phase systems with unknown and/or time varying delay, Automatica, 35:1417-1426.

[28] Mitsukura, Y., Yamamoto, T., Kaneda, M., Fukumi, M., Akamatsu, N. and Shah, S.L. (2000). Design and experimental evaluation of self-tuning PID controller using evolutionary computation, Preprints Proc. PID '00: IFAC Workshop on digital control (Past, present and future of PID control), 642-647.

[29] Chen, H.-F. and Zhang, J.-F. (1990). Identification and adaptive control for systems with unknown orders, delay and coefficients. IEEE Transactions on Automatic Control, 35: 866-877.

[30] Zhang, J.-F. and Chen, H.-F. (1990). Identification of coefficients, orders and time-delay for ARMAX systems. Proc.11th IFAC World Congress on Automatic Control, 3:129-134.

[31] Chen, H.-F., Guo, L. and Zhang, J.-F. (1991). Identification and adaptive control for ARMAX systems, Lecture Notes in Control and Information Sciences, 161:216-241.

[32] Keviczky, L. and Banyasz, Cs. (1992). An adaptive PID regulator based on time delay estimation. Proc. 31st Conference on Decision and Control, 3243-3248.

[33] Banyasz, Cs. and Keviczky, L. (1999). An adaptive and iterative scheme for PID autotuning based on design formulae, Proc. American Control Conference, 6:4358-4362.

[34] Gurubasavaraj, K.H. and Brogan, W.L. (1983). Identification of time delay in multiple input, multiple output discrete systems. Proc. American Control Conference, 1253-1254.

[35] Song, W.-Z. and Xu, Y. (1985). Structure and parameter identification for a kind of multivariable linear systems with unknown time delays. Proc. IFAC Identification and System Parameter Estimation Conference, 793-798.

[36] Prager, D.L. and Wellstead, P.E. (1980). Multivariable pole assignment self-tuning regulators, Proceedings of the IEE, Part D, 128:9-18.

[37] Vogel, E.F. and Edgar, T.F. (1988). An adaptive pole placement controller for chemical processes with variable dead time, Computers and Chemical Engineering, 12:15-26.

[38] Chien, I.-L., Seborg, D.E. and Mellichamp, D.A. (1985b). A self tuning controller for systems with unknown or time varying time delays, International Journal of Control, 42:949-964.

[39] Mizuno, N. and Fujii, S. (1983). Discrete time multivariable adaptive control for non-minimum phase plants with unknown dead time, Proc. IFAC Adaptive Systems in Control and Signal Processing, 363-368.

[40] Gao, R. and O'Dwyer, A. (2001). Comparison of two B-polynomial methods: application to the identification of time delayed processes, Proc. Irish Signals and Systems Conference, 105-111.

[41] Wood, R.K. and Berry, M.W. (1973). Terminal composition control of a binary distillation column, Chemical Engineering Science, 28:1707.

[42] Readle, J.C. (1993). Teaching system identification to undergraduate students, International Journal of Electrical Engineering Education, 30:44-50.

[43] Armstrong, B. (1997). A controls laboratory program with an accent on system identification, Proc. American Control Conference, 3166-3170.

[44] Rivera, D.E. and Flores, M.E. (2000). Beyond step testing and process reaction curves: introducing meaningful system identification concepts in the undergraduate chemical engineering curriculum, Proc. SYSID Conference.

[45] Keviczky, L. and Banyasz, Cs. (1988). A completely adaptive PID regulator, Proc. IFAC Identification and System Parameter Estimation Conference, 89-95.

[46] Glentis, G.-O. A. and Kalouptsidis, N. (1992). Efficient order recursive algorithms for multichannel least squares filtering. IEEE Transactions on Signal Processing, 40:1354-1374.

[47] Ferretti, G., Maffezzoni, C. and Scattolini, R. (1991). Recursive estimation of time delay in sampled systems. Automatica, 27:653-661.

[48] Kim Y.-C., Chung C.-S. and Yang H.-S. (1987). A new algorithm for the estimation of variable time delay of discrete systems. J. Korea Inst. Electron. Eng. (South Korea), 24:52-59.

[49] Dumont, G.A., Elnagger, A. and Elshafei, A. (1993). Adaptive predictive control of systems with time-varying time delay. International Journal of Adaptive Control and Signal Processing, 7:91-101.

[50] Xia, L., Moore, J.B. and Gevers, M. (1987). On adaptive estimation and pole assignment of overparametrized systems. International Journal of Adaptive Control and Signal Processing, 1:143-160.

[51] Xia, L. and Moore, J.B. (1989). Recursive identification of overparameterised systems. IEEE Transactions on Automatic Control, 34:327-331.

[52] Lee, T.H. and Hang, C.C. (1985). A performance study of parameter estimation schemes for systems with unknown dead time. Proc. American Control Conference, $1: 512-516$.

[53] Vogel, E.F. and Edgar, T.F. (1982). Application of an adaptive pole-zero placement controller to chemical processes with variable dead time. Proc. American Control Conference, 536-544.

[54] Seborg, D.E., Edgar, T.F. and Shah, S.L. (1986). Adaptive control strategies for process control: a survey. AIChE Journal, 32:881-913.

[55] Isermann, R. (1989). Digital Control Systems Volume 1. Fundamentals, Deterministic Control, 2nd Revised Edition, Springer- Verlag.

[56] Terrett, P. and Downing, C.J. (1993). System identification and modelling on the TMS320C25 fixed-point processor using the delta operator, Proc. Irish Colloquium on DSP and Control, 239-247.

[57] O'Dwyer, A. (2004). System identification using the delta operator, Poster presentation, Information Technology and Telecommunications (IT\&T) Conference. 